\title{
Seletividade de formulações e doses de 2,4-D para cultivares de amendoim
}

Submetido-03 Ago. 2021 Aprovado-15 ago. 2021 Publicado - 30 set. 2021

http://dx.doi.org/10.52755/sas.v.2i(edesp2)155

Universidade Estadual Paulista "Júlio de Mesquita Filho", UNESP, Campus Jaboticabal, Departamento de Biologia Aplicada a Agropecuária. E-mail: tsouza_oliveira@hotmail.com.

Willians César Carrega (iD Universidade Estadual Paulista "Júlio de Mesquita Filho", UNESP, Jaboticabal, São Paulo. E-mail: willianscesar@hotmail.com.

Anne Elise Cesarin (iD

Universidade Estadual Paulista "Júlio de Mesquita Filho", UNESP, Jaboticabal, São Paulo. E-mail: annecesarin@gmail.com.

Arthur Arrobas Martins Barroso (D)

Universidade Estadual Paulista "Júlio de Mesquita Filho", UNESP, Jaboticabal, São Paulo. E-mail: arthuragro07@hotmail.com.

Pedro Luis da Costa Aguiar Alves (D) Universidade Estadual Paulista "Júlio de Mesquita Filho", UNESP, Jaboticabal, São Paulo. E-mail: pl.alves@unesp.br.

\section{RESUMEN}

A interferência de plantas daninhas na cultura do amendoim é um dos principais fatores limitantes do crescimento, desenvolvimento e produtividade da cultura. Em virtude disso, a busca por alternativas para o controle de plantas daninhas é necessária para realização do manejo da cultura. Desta forma, objetivou-se avaliar os efeitos fitotóxicos de diferentes formulações comerciais e doses de 2,4-D em genótipos de amendoim rasteiro. Os genótipos estudados foram: IAC-OL3, IAC-870, IAC-505 e IAC-OL4. Para isso, o experimento foi conduzido em delineamento inteiramente casualizado, com quatro repetições, em esquema fatorial $2 \times 8$ (formulações comerciais $x$ doses). As doses isoladas de 2,4-D aplicadas foram: 0, 228, 456, 910 e $1370 \mathrm{~g}$ e.a. $\mathrm{ha}^{-1}$, aos 25 dias após a emergência (DAE), e as sequenciais foram: 114+228, 228+228, 228+456 g a.i. ha-1, aplicadas aos 25 e 40 DAE. A fitointoxicação foi avaliada visualmente aos 7, 14, 21, 28 e 35 dias após a aplicação dos tratamentos (DAA). Aos 35 DAA foram determinados o comprimento da haste principal, número de ramos primários, comprimento do ramo primário mais desenvolvido e massa seca da parte aérea, raiz e total. Constatou-se que as plantas submetidas às maiores doses apresentaram intoxicação inicial alta. Além disso, observou-se comportamento morfológico diferencial entre os genótipos. Independente do tipo de formulação do herbicida (sal de colina e sal de amina), a aplicação de 2,4-D nas doses 228, 456, 114+128; 228+228 g e.a. ha-1 não foram fitotóxicas. Por outro lado, as doses 1370; 910 e 228+456 g e.a. ha-1 foram fitotóxicas para as cultivares estudados.

Palavras-chave: Arachis hypogaea L.; Herbicidas; Interferência; Intoxicação; Plantas daninhas.

Selectivity of 2,4-D formulations and doses for peanut genotypes

ABSTRACT

Este es un trabajo de acceso abierto y distribuido bajo los Términos de Creative Commons Attribution Attribution-NonCommercial-ShareAlike 4.0 International. 
Weed interference in peanut is one of the main limiting factors of plant growth, development and productivity. Based on this assumption, to develop alternative weed control is necessary to weed management. The objective of this study was to evaluate the phytotoxic effects of different formulations and doses of 2,4-D on runner peanut genotypes. The genotypes evaluated were: IAC OL3, IAC 870, IAC 505 and IAC OL4. The experiment was carried out in a completely randomized design, with four replicates, arranged in a $2 \times 8$ factorial scheme (herbicide formulation $x$ doses). The single doses of 2,4-D applied were: $0,228,456,910$ and $1370 \mathrm{~g}$ a.i. $\mathrm{ha}^{-1}$ at 25 days after emergence (DAE), and the sequential doses were: $114+228,228$ $+228,228+456 \mathrm{~g}$ a.e. $\mathrm{ha}^{-1}$ applied at 25 and 40 DAE. The phytotoxic effects were evaluated at 7, 14, 21, 28 and 35 days after the treatment application (DAT). At 35 DAT were determined the main stem length, the number of primary branches, the length of the most developed primary branch and the dry mass of shoot and root. It was verified that the plants submitted to the highest doses presented high initial intoxication. In addition, was observed differential morphological behavior among the genotypes. Regardless of the type of 2,4-D formulation (choline salt and amine salt), the application of 2,4-D at 228, 456, $114+128,228+228 \mathrm{~g}$ a. e. $\mathrm{ha}^{-1}$ were not phytotoxic. The doses at 1370; 910 and $228+456 \mathrm{~g}$ a. e. ha ${ }^{-1}$ were phytotoxic to the genotypes.

Keywords: Arachis hypogaea L.; Herbicides. Intoxication; Weeds; Interference.

\title{
Selectividad de formulaciones y dosis de 2,4-D para cultivares de maní
}

\begin{abstract}
RESUMO
La interferencia de malezas en los cultivos de maní es uno de los principales factores limitantes para el crecimiento, desarrollo y productividad de los cultivos. Por tanto, la búsqueda de alternativas para el control de malezas es necesaria para llevar a cabo el manejo del cultivo. Así, el objetivo fue evaluar los efectos fitotóxicos de diferentes formulaciones comerciales $y$ dosis de 2,4-D sobre genotipos de maní rastrero. Los genotipos estudiados fueron: IAC 505, IAC OL3, IAC OL4 y Linaje 870. El experimento se realizó en un diseño completamente al azar, con cuatro repeticiones, en un esquema factorial $2 \times 8$ (formulaciones comerciales $x$ dosis). Las dosis de 2,4-D aislado aplicadas fueron: 0, 228, 456, 910 y $1370 \mathrm{~g}$ a.e. $h^{-1}$, a los 25 días después de la emergencia (DDE), y las secuenciales fueron: $114+228,228$ + 228, 228 + $456 \mathrm{~g}$ a.e. ha $^{-1}$, aplicado a los 25 y 40 DDE. La fitointoxicación se evaluó visualmente a los 7, 14, 21, 28 y 35 días después de la aplicación de los tratamientos (DDA). A los 35 DDA se determinó la longitud del tallo principal, el número de ramas primarias, la longitud de la rama primaria más desarrollada y la masa seca del dosel, raíz y total. Se observó que las plantas sometidas a las dosis más altas presentaban una intoxicación inicial elevada. Además, se observó un comportamiento morfológico diferencial entre genotipos. Independientemente del tipo de formulación herbicida (sal de colina y sal de amina), la aplicación de 2,4-D en dosis 228, 456, 114 + 128; 228 + $228 \mathrm{~g}$ a.e. ha ${ }^{-1}$ no fueron fitotóxicos. Por otro lado, dosis 1370; 910 y 228 + $456 \mathrm{~g}$ a.e. $\mathrm{ha}^{-1}$ fueron fitotóxicos para los cultivares estudiados.
\end{abstract}

Palabras clave: Arachis hypogaea L.; Herbicidas; Interferencia; Intoxicación; Malezas.

\section{Introdução}

O amendoim (Arachis hypogaea L.) destaca-se por ser uma leguminosa muito utilizada na alimentação humana e animal. Considerada uma das principais culturas oleaginosas, na última safra apresentou uma produção mundial de cerca de 94,4 milhões de toneladas (USDA, 2021).

Brasil é responsável por apenas 1\% desse total produzido. Contudo, o país tem potencial para aumentar a produção interna e difundir a exportação do produto, principalmente pela ampliação da área de cultivo. No Brasil, a 
principal região produtora de amendoim é a sudeste, sendo o Estado de São Paulo responsável por mais de $90 \%$ do total produzido (CONAB, 2021a). Deste total, quase toda a produção de amendoim advém de áreas em sucessão com a cana-de-açúcar (CONAB, 2021b).

Áreas utilizadas em rotação de culturas apresentam grandes problemas com ocorrência de plantas daninhas que podem reduzir drasticamente a produtividade e qualidade da produção. As plantas daninhas causam interferência no crescimento, desenvolvimento e, consequentemente, redução na produtividade das culturas, decorrente principalmente da competição por água, luz e nutrientes (PITELLI, 1985), afetando também a qualidade dos grãos/sementes da cultura, além de liberar substância alelopáticas e hospedar doenças e pragas (PITELLI, 1987). Em amendoim, essa interferência tem causado perdas na produtividade da cultura que variam entre 31 e 92\% (NEPOMUCENO et al., 2007; AGOSTINHO et al., 2006; YAMAUTI et al., 2010).

Entre os diversos manejos realizados no controle de plantas daninhas, a utilização de herbicidas é uma prática comum na agricultura, sendo a adoção de controle químico o mais utilizado. Segundo Alvino et al. (2011), esse manejo apresenta maiores vantagens por ser mais eficiente $e$ econômico para controle das plantas daninhas. Contudo, para a cultura do amendoim, existem apenas seis herbicidas registrados junto ao Ministério da Agricultura, Pecuária e Abastecimento (MAPA) destinados ao controle de plantas daninhas em pré e pós-emergência, dentre eles, alachlor, pendimethalin, trifluralin (pré-emergentes) e bentazon, imazapic e quizalofop-p-ethyl (pós-emergentes) (RODRIGUES e ALMEIDA, 2011). Diante da escassez de herbicidas, no ano de 2015 o amendoim foi incluído junto ao MAPA como uma Cultura com Suporte Fitossanitário Insuficiente (CSFI) (MAPA, 2015).

O 2,4-Diclorofenoxiacético ácido (2,4-D) foi o primeiro herbicida sintético desenvolvido e teve o início de sua comercialização na década de 40. Sendo um herbicida sistêmico e seletivo em controle de plantas eudicotiledôneas, apresentando alta eficácia no controle de plantas daninhas 
conhecidas como espécies de folhas largas. Classificado como um herbicida mimetizador de auxinas pertencente ao grupo dos ácidos fenoxicarboxílicos (OLIVEIRA JÚNIOR et al., 2007; SONG, 2014). Por ser um herbicida muito utilizado, empresas estão sempre investindo em novas formulações como aconteceu com o sal de colina de 2,4-D (ANONYMOUS, 2015; LI et al., 2013). Esta nova formulação apresenta maior estabilidade e menor chance de volatilização do que os ésteres e amina (SOSNOSKIE et al., 2015).

Os compostos da formulação de um herbicida podem influenciar na absorção e translocação do produto nas plantas e, consequentemente, poderão causar efeitos fitotóxicos, comprometer o crescimento, desenvolvimento e produtividade das culturas (PETERSON et al., 2016).

Dessa forma, há a necessidade de estudos visando avaliar o comportamento das plantas submetidas às formulações de 2,4-D, com o intuito de selecionar formulações que controlem as plantas daninhas, sem afetar às culturas. Diante disso, os efeitos fitotóxicos de diferentes formulações e doses de 2,4-D, foram avaliados em cultivares de amendoim.

\section{Material e métodos}

Os experimentos foram desenvolvidos em ambiente aberto, sem restrição de água, utilizando-se como recipientes vasos plásticos com capacidade para 2,5 L preenchidos com substrato composto por mistura de solo e areia $(2: 1 \mathrm{v} / \mathrm{v})$. O solo utilizado é classificado como Latossolo Vermelho distrófico, de textura média (EMBRAPA, 2013), cujos resultados da análise química estão descritos na Tabela 1.

Tabela 1. Resultado da análise química do solo utilizado como substrato em mistura com areia.

\begin{tabular}{cccccccccc}
\hline & & & & \multicolumn{3}{c}{$\mathrm{H}+\mathrm{Al}$} & Soma Bases & Sat. \\
\hline $\mathrm{pH}$ & M.O. & P. resina & $\mathrm{K}$ & $\mathrm{Ca}$ & $\mathrm{Mg}$ & $\mathrm{SMP}$ & S.B. & $\mathrm{T}$ & Bases \\
\hline $\mathrm{CaCl}_{2}$ & $\mathrm{~g} \mathrm{dm}^{-3}$ & $\mathrm{mg} \mathrm{dm}^{-3}$ & & & \multicolumn{3}{c}{$\mathrm{mmol} . \mathrm{dm}^{-3}$} & & \\
\hline 5,6 & 13 & 34 & 1,8 & 24 & 6 & 15 & 31,8 & 46,8 & 68 \\
\hline
\end{tabular}

$\mathrm{pH}$, potencial hidrogeniônico; $\mathrm{CaCl}_{2}$, Cloreto de Cálcio; M.O., matéria orgânica; P., fósforo; K, potássio; $\mathrm{Ca}$, cálcio; Mg, magnésio; $\mathrm{H}$ + Al, acidez potencial pelo método SMP; Sat., saturação; g, gramas; $\mathrm{dm}^{-3}$, decímetro cúbico; mmol., milimol. 
O estudo foi conduzido em delineamento experimental inteiramente casualizado, com quatro repetições. Em esquema fatorial $2 \times 8$, tendo como tratamentos, duas formulações de 2,4-D (sal de amina e sal de colina) submetidos a sete doses $(228,456,910,1370,114+228,228+228,228+456$ g e.a. ha-1) e um tratamento testemunha (sem aplicação). Para cada genótipo, foi considerado um experimento independente.

Foram utilizadas quatro cultivares de amendoim rasteiro (IAC-OL3, IAC-870, IAC-505 e IAC-OL4) cedidos pelo Instituto Agronômico de Campinas. Em cada vaso foi semeada uma cultivar, depositando-se sete sementes a $3 \mathrm{~cm}$ de profundidade. As sementes foram previamente tratadas com o inseticida thiamethoxam e o fungicida carboxin + thiram.

Após sete dias da emergência das plantas, realizou-se desbaste, mantendo-se duas plantas por vaso. Como tratamento preventivo ao ataque de insetos-pragas e doenças fúngicas, aos 35 dias após a semeadura e semanalmente durante todo o período experimental, realizou-se aplicação do inseticida thiamethoxam (200 mL ha-1) e do fungicida pyraclostrobin (600 $m L h^{-1}$ ).

A aplicação das formulações de 2,4-D foi realizada aos 25 dias após emergência (DAE) para os tratamentos com dose única e, aos 25 e $40 \mathrm{DAE}$ para a aplicação sequencial. Para cada aplicação foi utilizado pulverizador costal pressurizado por $\mathrm{CO}_{2}$, munido de barras com quatro pontas tipo leque (TTJ60-11002), espaçados em 0,5m entre eles, operando a 2,3 $\mathrm{kgf.cm}^{-2}$ de pressão e trabalhando com volume de calda correspondente a $200 \mathrm{~L} \mathrm{ha}^{-1}$, com deslocamento a $1 \mathrm{~m} \mathrm{~s}^{-1}$, barra a 0,5 metro de altura em relação ao alvo.

Para avaliar a intoxicação causada pelas formulações de 2,4-D nas plantas, foi realizada avaliações visuais aos $7,14,21,28$ e 35 dias após a aplicação (DAA) dos tratamentos em pós-emergência, utilizando-se uma escala de notas proposta pela European Weed Research Council (EWRC, 1964), na qual a nota 1 (um) representou a ausência de sintomas e a 9 (nove) a morte das plantas. 
Aos 35 DAA foram realizadas avaliações morfológicas. Para isso, foram realizadas medições do comprimento da haste principal, número de ramos primários e comprimento do ramo primário mais desenvolvido das plantas de amendoim. Após essas avaliações, as plantas foram separadas em parte área e raiz e foram acondicionadas em estufa com circulação forçada de ar a $65^{\circ} \mathrm{C}$ por 76 horas, até obtenção da massa constante. Em seguida, determinou-se a massa seca da parte aérea, radicular e total.

Os resultados obtidos foram submetidos à análise de variância e as médias comparadas pelo teste de Tukey a 5\% de probabilidade. Quando significativas para a fitointoxicação, as médias foram submetidas à análise de regressão pelo modelo polinomial de segundo grau $\left(y=a x^{2}+b x+c\right)$, no qual $\mathrm{Y}$ refere-se à nota de fitointoxicação $\mathrm{e} X$ refere-se aos dias de avaliação após a aplicação dos produtos, utilizando o software estatístico AgroEstat (BARBOSA; MALDONADO JUNIOR, 2015).

\section{Resultados e discussão}

As cultivares de amendoim (IAC OL3, IAC 870, IAC 505 e IAC OL4) apresentaram crescimento inicial anormal, independente da dose ou formulação de 2,4-D. Entre os sintomas, se observou epinastia foliar, inchaço nos tecidos, leve necrose, torção da haste principal e dos ramos laterais do amendoim, poucas horas após a aplicação do 2,4-D, sintomas característicos do herbicida utilizado algumas horas após a aplicação. Inicialmente, todas as doses causaram efeito tóxico, variando de leve a muito forte (EWRC, 1984), dependendo da dose e da aplicação (única ou sequencial) (Figura 1).

As maiores notas de intoxicação foram constatadas nas plantas de todos as cultivares submetidas às maiores doses (1370 e $910 \mathrm{~g}$ e.a. ha-1) do 2,4-D até os 21 DAA, independente da formulação (Tabela 2). Entretanto, após isso, as plantas apresentaram alto nível de recuperação e esse efeito foi reduzido ao longo do tempo, sendo considerada aos 35 DAA uma intoxicação leve a mediana, segundo EWRC (1984). 

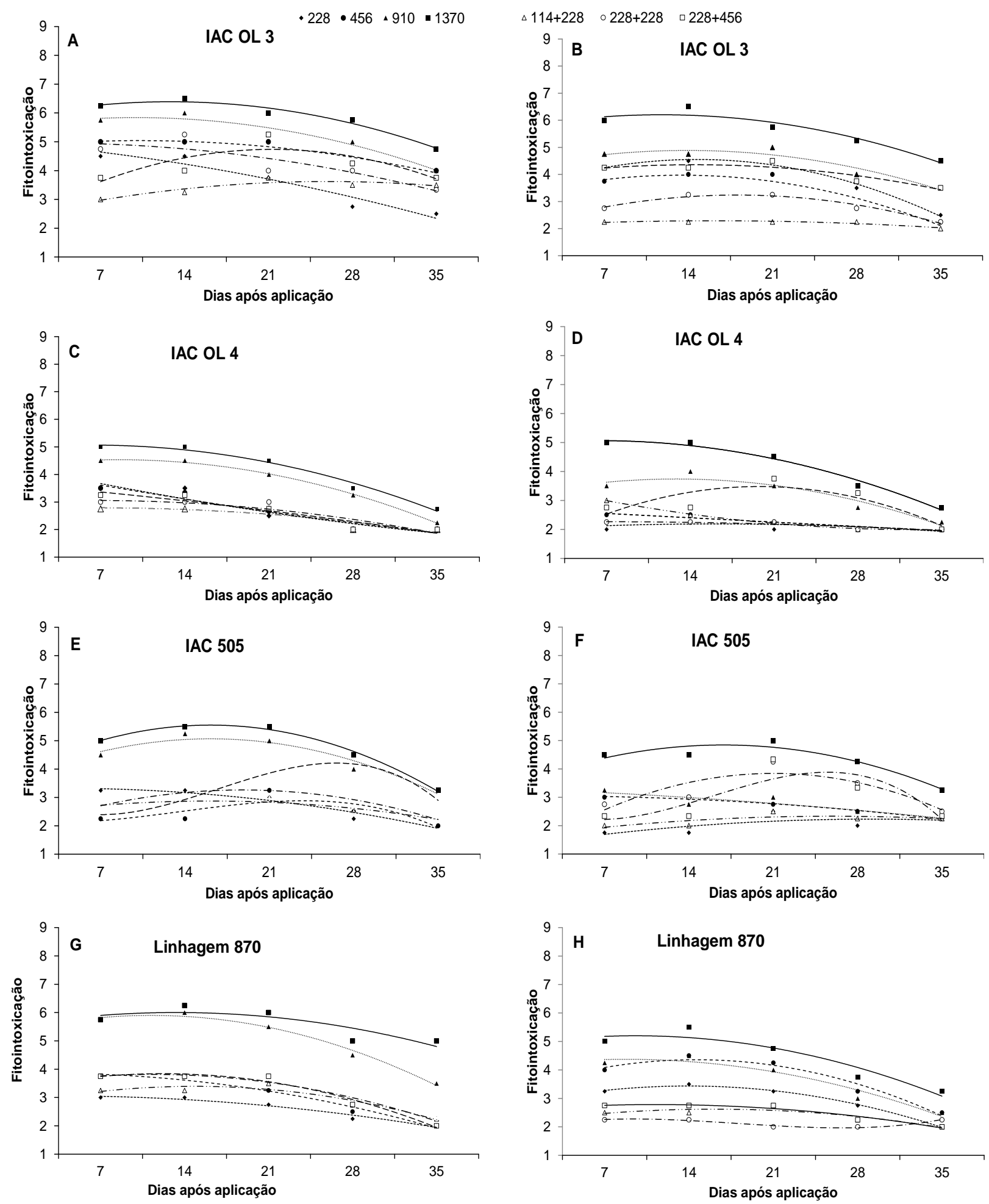

Figura 1. Fitointoxicação de cultivares de amendoim submetidas às formulações e doses de 2,4-D. A, C, E, G - aplicação de 2,4-D (sal de colina); B, D, F, H - aplicação de 2,4-D (sal de amina). 
Tabela 2. Parâmetros da regressão estimados pela equação polinomial de segundo grau para dois cultivares de amendoim submetidas às formulações e doses de 2,4-D.

\begin{tabular}{|c|c|c|c|c|}
\hline Doses (g e.a./ha) & Sal de colina & $\mathbf{R}^{2}$ & Sal de amina & $\mathbf{R}^{2}$ \\
\hline \multicolumn{5}{|c|}{ IAC OL3 } \\
\hline 228 & $y=-0,05 x^{2}-0,25 x+4,95$ & 0,94 & $y=-0,04 x^{2}+0,16 x+2,10$ & 0,86 \\
\hline 456 & $y=-0,09 x^{2}+0,26 x+4,85$ & 0,91 & $y=-0,20 x^{2}+0,75 x+3,25$ & 0,91 \\
\hline 910 & $y=-0,14 x^{2}+0,41 x+5,55$ & 0,95 & $y=-0,16 x^{2}+0,64 x+4,25$ & 0,90 \\
\hline 1370 & $y=-0,16 x^{2}+0,59 x+5,85$ & 0,97 & $y=-0,16 x^{2}+0,54 x+5,75$ & 0,93 \\
\hline $114+228$ & $y=-0,09 x^{2}+0,66 x+2,40$ & 0,82 & $y=-0,25 x^{2}+1,05 x+3,45$ & 0,98 \\
\hline $228+228$ & $y=-0,08 x^{2}+0,06 x+4,95$ & 0,79 & $y=-0,18 x^{2}+0,92 x+2,05$ & 0,96 \\
\hline $228+456$ & $y=-0,27 x^{2}+1,63 x+2,25$ & 0,65 & $y=-0,11 x^{2}+0,44 x+3,90$ & 0,83 \\
\hline \multicolumn{5}{|c|}{ IAC OL4 } \\
\hline 228 & $y=0,04 x^{2}-0,66 x+4,30$ & 0,89 & $y=-0,04 x^{2}+0,16 x+2,00$ & 0,61 \\
\hline 456 & $y=0,02 x^{2}-0,58 x+4,17$ & 0,93 & $y=0,02 x^{2}-0,58 x+4,17$ & 0,93 \\
\hline 910 & $y=-0,16 x^{2}+0,39 x+4,30$ & 0,99 & $y=-0,16 x^{2}+0,59 x+3,20$ & 0,92 \\
\hline 1370 & $y=-0,14 x^{2}+0,26 x+4,95$ & 0,98 & $y=-0,14 x^{2}+0,26 x+4,95$ & 0,98 \\
\hline $114+228$ & $y=-0,05 x^{2}+0,10 x+2,75$ & 0,81 & $y=-0,07 x^{2}+0,68 x+3,60$ & 0,99 \\
\hline $228+228$ & $y=-0,07 x^{2}+0,13 x+3,00$ & 0,81 & $y=-0,02 x^{2}+0,03 x+2,25$ & 0,81 \\
\hline $228+456$ & $y=-0,02 x^{2}-0,27 x+3,65$ & 0,90 & $y=-0,29 x^{2}+1,61 x+1,20$ & 0,73 \\
\hline \multicolumn{5}{|c|}{ IAC 505} \\
\hline 228 & $y=-0,07 x^{2}+0,08 x+3,30$ & 0,94 & $y=-0,05 x^{2}+0,45 x+1,30$ & 0,46 \\
\hline 456 & $y=-0,20 x^{2}+1,15 x+1,15$ & 0,59 & $y=-0,04 x^{2}+0,01 x+3,05$ & 0,98 \\
\hline 910 & $y=-0,27 x^{2}+1,23 x+3,65$ & 0,94 & $y=-0,02 x^{2}-0,12 x+3,30$ & 0,82 \\
\hline 1370 & $y=-0,32 x^{2}+1,48 x+3,85$ & 0,99 & $y=-0,23 x^{2}+1,12 x+3,50$ & 0,90 \\
\hline $114+228$ & $y=-0,09 x^{2}+0,41 x+2,40$ & 0,82 & $y=-0,06 x^{2}+0,40 x+1,60$ & 0,55 \\
\hline $228+228$ & $y=-0,20 x^{2}+1,05 x+1,85$ & 0,84 & $y=-0,32 x^{2}+1,93 x+0,95$ & 0,75 \\
\hline $228+456$ & $y=-0,30 x^{2}+2,05 x+0,45$ & 0,60 & $y=-0,36 x^{2}+2,24 x+0,13$ & 0,59 \\
\hline \multicolumn{5}{|c|}{ IAC 870} \\
\hline 228 & $y=-0,05 x^{2}+0,05 x+3,05$ & 0,97 & $y=-0,16 x^{2}+0,64 x+2,80$ & 0,99 \\
\hline 456 & $y=-0,09 x^{2}+0,06 x+3,85$ & 0,98 & $y=-0,23 x^{2}+0,97 x+3,35$ & 0,96 \\
\hline 910 & $y=-0,21 x^{2}+0,69 x+5,35$ & 0,99 & $y=-0,14 x^{2}+0,36 x+4,15$ & 0,94 \\
\hline 1370 & $y=-0,13 x^{2}+0,48 x+5,55$ & 0,74 & $y=-0,16 x^{2}+0,44 x+4,90$ & 0,91 \\
\hline $114+228$ & $y=-0,14 x^{2}+0,61 x+2,75$ & 0,91 & $y=-0,09 x^{2}+0,41 x+2,15$ & 0,82 \\
\hline $228+228$ & $y=-0,14 x^{2}+0,46 x+3,45$ & 0,94 & $y=0,05 x^{2}-0,35 x+2,60$ & 0,62 \\
\hline $228+456$ & $y=-0,18 x^{2}+0,62 x+3,30$ & 0,97 & $y=-0,07 x^{2}+0,23 x+2,60$ & 0,94 \\
\hline
\end{tabular}


A aplicação sequencial na maior dose (228+456 g e.a. ha-1) apresentou comportamento semelhante ao observado para a aplicação isolada nas maiores doses (Figura 1). Blanchett et al. (2017) observaram danos semelhante aos constatados no presente estudo e mencionaram que os sintomas foram transitórios e, ao final do período experimental, as injúrias nas plantas desapareceram. Segundo esses autores, o dano do 2,4-D na dose de $1066 \mathrm{~g}$ e.a. $\mathrm{ha}^{-1}$ foi $10 \%$ maior quando comparado com a testemunha.

Pesquisas anteriores mostraram que o impacto no rendimento do amendoim submetido a herbicidas não seletivos varia significativamente dependendo do herbicida e da dose (LEON et al., 2014). Johnson et al. (2012), testando o 2,4-D na dose de $540 \mathrm{~g} \mathrm{ha}^{-1}$, observaram que em plantas de amendoim as injúrias variaram entre 30 e 40\% após uma e duas semanas da aplicação, respectivamente. Leon et al. (2014), testando doses de 2,4-D, constataram que as maiores injúrias foram causadas pelas maiores doses (560 e 1120 g e.a. ha ${ }^{-1}$ ) com 15 e 35\% de danos, respectivamente. Nas demais doses $\left(70,140\right.$ e 280, g e.a. ha $\left.{ }^{-1}\right)$, os autores não observaram diferenças. Segundo esses autores, 0 amendoim apresentou alto nível de tolerância ao uso de 2,4-D. Esses dados corroboram os observados no presente estudo, que mostram que nas menores doses, aos $35 \mathrm{DAA}$, as injúrias no amendoim foram consideradas baixas, demonstrando, assim, que o uso de 2,4-D, independente da formulação, pode ser uma alternativa viável para o controle de plantas daninhas nas áreas de amendoim.

Entre as cultivares foi possível constatar que o IAC OL3 foi visualmente mais sensível às aplicações de 2,4-D, apresentando as maiores injúrias quando comparado as demais cultivares, independente da formulação (Figuras 1A e 1B). Entre as formulações de 2,4-D, constatou-se que o sal de colina proporcionou fitointoxicação inicial maior, quando comparado com o sal de amina. Entretanto, verificou-se que esse efeito foi reduzido ao longo do tempo e, até os 35 DAA, essa diferença não foi mais constatada (Figura 1). 
As injúrias causadas pelo 2,4-D geralmente ocorrem durante as primeiras horas após aplicação de herbicidas auxínicos (1-2 h). Inicialmente há a estimulação das plantas, por meio da ativação de alguns processos metabólicos, tais como a estimulação da biossíntese de etileno, por meio da indução da síntese de 1-aminociclopropano-1-carboxílico (ACC) no tecido dos ramos (1-2 h), seguido de crescimento anormal (desregulado) (3-4 h), como os observados no presente estudo. Após 24 h da aplicação, ocorre a redução da área foliar e da interceptação da radiação.

Simultaneamente, ocorre o fechamento estomático e, consequentemente, redução na atividade transpiratória, assimilação de carbono e formação de amido. Diante disso, há uma superprodução de espécies reativas de oxigênio (ERO) (GROSSMANN, 2010). Como consequência do aumento das ERO, ocorre a redução na produção de aminoácidos, amidos, ácidos nucleicos e outros metabolitos primários fundamentais para 0 crescimento e desenvolvimento das plantas (GROSSMANN, 2010; PETERSON et al., 2016).

Nas avaliações de crescimento e desenvolvimento das plantas, verificou-se que para a altura (Tabela 3) as plantas da linhagem 870 foram mais sensíveis às aplicações em doses únicas. Quando essas plantas foram submetidas a doses superiores a $228 \mathrm{~g}$ e.a.ha-1 houve menor crescimento da haste principal. Para o IAC OL3, mesmo apresentando os maiores sintomas de intoxicação, quando as plantas foram submetidas às aplicações de 2,4-D, não foram constatadas diferenças em relação à altura das plantas (Tabela 3), comprimento dos ramos primários (Tabela 5) e número de ramos primários (Tabela 6). Para as demais cultivares (IAC 505 e IAC OL4), constatou-se interação entre as formulações e doses testadas para a altura das plantas (Tabela 4).

Com base no desdobramento das interações para a altura das plantas (Tabela 4), verificou-se que entre as doses para cada formulação de 2,4-D, as aplicações sequenciais de sal de colina e sal de amina proporcionaram maior altura das plantas da cultivar IAC 505. Para a aplicação isolada do sal de colina, constatou-se que o uso da menor dose 
(228 g e.a.ha-1) proporcionou menor altura da cultivar IAC 505 e na dose (456 g e.a.ha-1) para a cultivar IAC OL4.

Para a aplicação isolada do sal de amina, constatou-se que a altura das cultivares IAC 505 e IAC OL4 foram reduzidas quando as plantas foram submetidas às maiores doses (910 e $1370 \mathrm{~g}$ e.a. ha $\left.{ }^{-1}\right)$. Esses dados corroboram os observados por Grossmann (2010), que verificou que em altas doses os herbicidas auxínicos, como o 2,4-D, podem causar intoxicação nas plantas, incluindo redução na área foliar, maior fragilidade e crescimento anormal.

Comparando as formulações para cada dose, verificou-se que a aplicação isolada na dose de $228 \mathrm{~g}$ e.a. ha ${ }^{-1}$ do sal de colina resultou em menor altura da cultivar IAC 505 e a dose única de $456 \mathrm{~g}$ e.a. ha-1 e a sequencial $228+228 \mathrm{~g}$ e.a. ha $^{-1}$ reduziram a altura da cultivar IAC OL4 (Tabela 4). Em baixas doses, os herbicidas auxínicos promovem 0 crescimento das plantas (GROSSMANN, 2010), mas esse efeito é dependente, além da dose, da espécie, do ingrediente ativo e do estágio de crescimento no momento da exposição (GUNSOLUS \& CURRAN, 1991). No presente estudo, inicialmente as cultivares IAC 505 e IAC OL4 apresentaram menor crescimento devido às injúrias causadas pelas doses das formulações de 2,4-D.

Contudo, esse menor crescimento não deve ser considerado como um fator negativo, uma vez que esse gasto de energia que seria destinado para o crescimento (i.e. altura), pode ser revertido para o desenvolvimento das plantas (i.e. ramos primários, secundários e número de nós) e, assim, proporcionar incrementos nos números de ginóforos que são responsáveis pela produção das vagens. Nesse sentido, o 2,4-D poderia ser considerado, nas menores doses, como um potencial regulador de crescimento, além de controlar as plantas daninhas existentes nas áreas de cultivo de amendoim. 
Tabela 3. Comprimento da haste principal $(\mathrm{cm})$ de amendoim 'IAC OL3' e linhagem 870 submetidas a diferentes formulações e doses de 2,4-D.

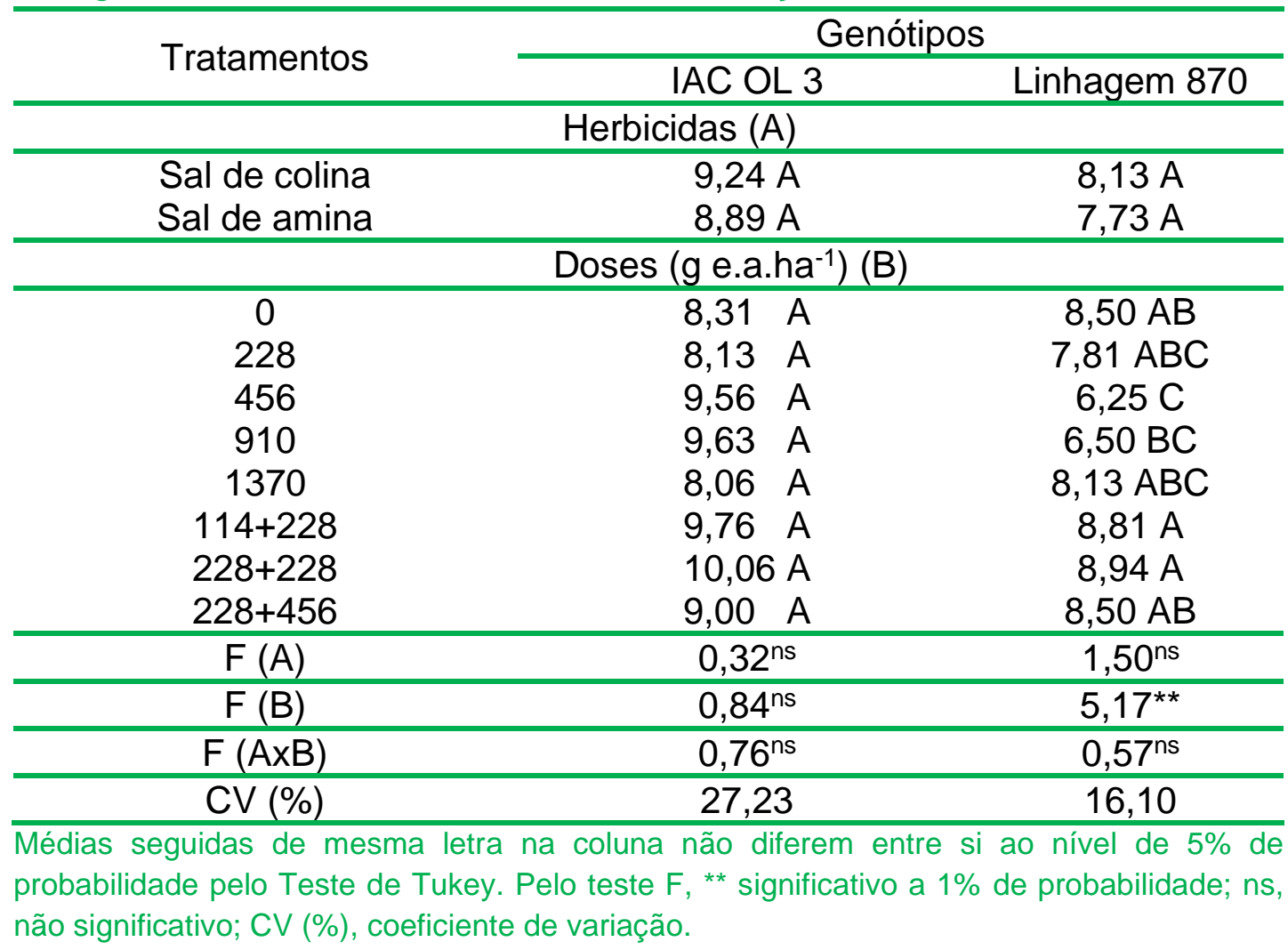

Tabela 4. Desdobramento entre os fatores herbicidas e doses para o comprimento da haste principal de plantas de amendoim 'IAC 505' e 'IAC OL4' submetidas a diferentes formulações e doses de 2,4-D.

\begin{tabular}{|c|c|c|c|c|c|c|}
\hline \multirow{3}{*}{$\begin{array}{c}\text { Doses } \\
\left(\mathrm{g} \text { e.a.ha }{ }^{-1}\right)\end{array}$} & \multicolumn{6}{|c|}{ Genótipos } \\
\hline & \multicolumn{3}{|c|}{ IAC 505} & \multicolumn{3}{|c|}{ IAC OL 4} \\
\hline & $\begin{array}{l}\text { Sal de } \\
\text { colina }\end{array}$ & $\begin{array}{l}\text { Sal de } \\
\text { amina }\end{array}$ & $F$ & $\begin{array}{l}\text { Sal de } \\
\text { colina }\end{array}$ & $\begin{array}{l}\text { Sal de } \\
\text { amina }\end{array}$ & $F$ \\
\hline 0 & $9,13 \mathrm{AB} a$ & $8,38 \mathrm{ABC} a$ & $0,86^{n s}$ & $10,13 \mathrm{Aa}$ & $9,75 \mathrm{AB}$ a & $0,20^{n s}$ \\
\hline 228 & $6,88 \mathrm{~B} \mathrm{~b}$ & $9,50 \mathrm{AB} a$ & 10,49 ** & $8,25 \mathrm{AB}$ a & $9,88 \mathrm{AB}$ a & 3,69 ns \\
\hline 456 & $7,75 \mathrm{AB}$ a & $8,75 \mathrm{ABC} a$ & $1,52 \mathrm{~ns}$ & 7,25 B b & $9,88 \mathrm{AB} a$ & $9,62^{* *}$ \\
\hline 910 & $8,00 \mathrm{AB}$ a & $6,88 \mathrm{C} \mathrm{a}$ & 1,93 ns & $8,75 \mathrm{AB} a$ & $8,63 \mathrm{~B}$ a & $0,02^{n s}$ \\
\hline 1370 & $7,75 \mathrm{AB} a$ & $7,13 \mathrm{BC}$ a & $0,59 \mathrm{~ns}$ & $8,88 \mathrm{AB} a$ & $8,13 \mathrm{~B} \mathrm{a}$ & $0,78^{\mathrm{ns}}$ \\
\hline $114+228$ & $9,75 \mathrm{~A} a$ & $9,75 \mathrm{~A} a$ & $0,00^{\mathrm{ns}}$ & $10,38 \mathrm{~A} a$ & $10,38 \mathrm{AB}$ a & $0,00^{n s}$ \\
\hline 228+228 & $9,88 \mathrm{~A} a$ & $9,63 \mathrm{AB} a$ & $0,10^{\mathrm{ns}}$ & $10,13 \mathrm{~A} b$ & $12,25 \mathrm{~A} \mathrm{a}$ & $6,31^{*}$ \\
\hline $228+456$ & $8,50 \mathrm{AB}$ a & $9,38 \mathrm{ABC}$ a & $1,17^{\mathrm{ns}}$ & $10,63 \mathrm{~A} a$ & $9,88 \mathrm{AB}$ a & $0,78^{\mathrm{ns}}$ \\
\hline $\mathrm{F}$ & $3,41^{\star \star}$ & $3,90^{* *}$ & ----- & $4,01^{* \star}$ & $4,20^{\star \star}$ & ----- \\
\hline
\end{tabular}

Médias seguidas de mesma letra maiúscula na coluna e minúscula na linha não diferem entre si ao nível de $5 \%$ de probabilidade pelo Teste de Tukey. Pelo teste $\mathrm{F}$, ${ }^{* *}$ significativo a $1 \%$ e *significativo a $5 \%$ de probabilidade; ns, não significativo; CV (\%), coeficiente de variação. 
Tabela 5. Comprimento dos ramos primários mais desenvolvidos de plantas de amendoim 'IAC OL3', 'IAC 505', 'IAC OL4' e linhagem 870 submetidas a diferentes formulações e doses de 2,4-D.

\begin{tabular}{cllll}
\hline \multirow{2}{*}{ Tratamentos } & \multicolumn{4}{c}{ Genótipos } \\
\cline { 2 - 5 } & IAC OL 3 & \multicolumn{4}{c}{ Linhagem 870 IAC 505 } & IAC OL 4 \\
\cline { 2 - 5 } Sal de colina & $14,80 \mathrm{~A}$ & $12,78 \mathrm{~A}$ & $13,82 \mathrm{~A}$ & $15,50 \mathrm{~A}$ \\
Sal de amina & $13,69 \mathrm{~A}$ & $12,61 \mathrm{~A}$ & $13,41 \mathrm{~A}$ & $15,63 \mathrm{~A}$ \\
\hline \multicolumn{5}{c}{ Doses $\left(\mathrm{g}\right.$ e.a.ha $\left.{ }^{-1}\right)(\mathrm{B})$} \\
\hline 0 & $14,46 \mathrm{~A}$ & $14,01 \mathrm{~A}$ & $14,50 \mathrm{AB}$ & $15,94 \mathrm{AB}$ \\
228 & $13,94 \mathrm{~A}$ & $13,56 \mathrm{AB}$ & $13,44 \mathrm{AB}$ & $15,63 \mathrm{AB}$ \\
456 & $14,25 \mathrm{~A}$ & $11,50 \mathrm{AB}$ & $13,50 \mathrm{AB}$ & $15,19 \mathrm{AB}$ \\
910 & $15,25 \mathrm{~A}$ & $11,44 \mathrm{~B}$ & $12,44 \mathrm{~B}$ & $14,69 \mathrm{~B}$ \\
1370 & $13,06 \mathrm{~A}$ & $11,63 \mathrm{~B}$ & $11,94 \mathrm{~B}$ & $14,13 \mathrm{~B}$ \\
$114+228$ & $14,69 \mathrm{~A}$ & $13,38 \mathrm{AB}$ & $15,00 \mathrm{~A}$ & $17,00 \mathrm{~A}$ \\
$228+228$ & $15,06 \mathrm{~A}$ & $13,14 \mathrm{AB}$ & $14,12 \mathrm{AB}$ & $16,38 \mathrm{AB}$ \\
$228+456$ & $13,25 \mathrm{~A}$ & $12,94 \mathrm{AB}$ & $14,00 \mathrm{AB}$ & $15,56 \mathrm{AB}$ \\
\hline $\mathrm{F}(\mathrm{A})$ & $2,79^{\mathrm{ns}}$ & $0,19^{\mathrm{ns}}$ & $1,06^{\mathrm{ns}}$ & $0,08^{\mathrm{ns}}$ \\
\hline $\mathrm{F}(\mathrm{B})$ & $0,72^{\mathrm{ns}}$ & $3,25^{\star *}$ & $3,12^{\star *}$ & $2,20^{* *}$ \\
\hline $\mathrm{F}(\mathrm{AxB})$ & $0,18^{\mathrm{ns}}$ & $1,35^{\mathrm{ns}}$ & $1,33^{\mathrm{ns}}$ & $1,88^{\mathrm{ns}}$ \\
\hline $\mathrm{CV}(\%)$ & 18,55 & 12,66 & 12,06 & 11,20 \\
\hline
\end{tabular}

Médias seguidas de mesma letra na coluna não diferem entre si ao nível de $5 \%$ de probabilidade pelo Teste de Tukey. Pelo teste $\mathrm{F},{ }^{* *}$ significativo a $1 \%$ de probabilidade; ns, não significativo; CV (\%), coeficiente de variação.

Para o comprimento dos ramos primários da cultivar IAC OL3 não se observou efeito negativo das doses. Para as demais cultivares (Linhagem 870, IAC 505 e IAC OL4), verificou-se que as plantas submetidas às maiores doses (1370 e $910 \mathrm{~g}$ e.a. ha ${ }^{-1}$ ) de 2,4-D apresentaram encurtamento dos ramos (Tabela 5). Contudo, não se observou redução para o número de ramos primários de todos as cultivares, quando comparadas com a testemunha (Tabela 6).

Esse efeito demonstra que as duas formulações poderiam realmente ser utilizadas como reguladores de crescimento visando reduzir o tamanho das plantas sem afetar o número de nós (local onde tem origem das flores, formação dos ginóforos (pegs) e posterior formação de vagens e sementes), e sem causar redução na produtividade dessa cultura.

Comparando o efeito das doses para as massas secas da parte aérea, raiz e total, observou-se que apenas a maior dose (1370 g e.a. ha-1 $)$ de 2,4D, independente da formulação, causou redução no acúmulo de massa da 
linhagem 870 quando comparada com a testemunha (Tabelas 7, 9 e 11). Para a massa seca da parte aérea da cultivar IAC OL3 (Tabela 8) e para a massa seca radicular da cultivar IAC 505 (Tabela 10), constatou-se interação entre os fatores doses versus formulações de 2,4-D. Para a cultivar IAC OL4 observou-se redução significativa no acúmulo de massa seca radicular apenas entre as doses, sendo a maior dose (1370 g e.a. ha $\left.{ }^{-1}\right)$ a mais prejudicial (Tabela 9).

Tabela 6. Número de ramos primários mais desenvolvidos de plantas de amendoim 'IAC OL3', 'IAC 505', 'IAC OL4' e linhagem 870 submetidas a diferentes formulações e doses de 2,4-D.

\begin{tabular}{|c|c|c|c|c|}
\hline \multirow{3}{*}{ Tratamentos } & \multicolumn{4}{|c|}{ Genótipos } \\
\hline & IAC OL 3 & Linhagem 870 & IAC 505 & IAC OL 4 \\
\hline & \multicolumn{4}{|c|}{ Herbicidas $(\mathrm{A})$} \\
\hline \multirow{3}{*}{$\begin{array}{l}\text { Sal de colina } \\
\text { Sal de amina }\end{array}$} & $6,65 \mathrm{~A}$ & $6,16 \mathrm{~A}$ & $5,39 \mathrm{~A}$ & $6,56 \mathrm{~A}$ \\
\hline & $6,45 \mathrm{~A}$ & $5,92 \mathrm{~A}$ & $5,39 \mathrm{~A}$ & $6,58 \mathrm{~A}$ \\
\hline & \multicolumn{4}{|c|}{ Doses (g e.a.ha-1) (B) } \\
\hline 0 & $6,88 \mathrm{~A}$ & $6,38 \mathrm{~A}$ & 5,44 & $6,00 \mathrm{~A}$ \\
\hline 228 & $6,88 \mathrm{~A}$ & $5,81 \mathrm{~A}$ & $5,81 \mathrm{~A}$ & $7,50 \mathrm{~A}$ \\
\hline 456 & $6,69 \mathrm{~A}$ & $5,88 \mathrm{~A}$ & $5,25 \mathrm{~A}$ & $6,56 \mathrm{~A}$ \\
\hline 910 & $6,19 \mathrm{~A}$ & $6,50 \mathrm{~A}$ & $5,50 \mathrm{~A}$ & $6,94 \mathrm{~A}$ \\
\hline 1370 & $6,25 \mathrm{~A}$ & $5,38 \mathrm{~A}$ & $5,19 \mathrm{~A}$ & $6,31 \mathrm{~A}$ \\
\hline $114+228$ & $6,31 \mathrm{~A}$ & $6,38 \mathrm{~A}$ & $5,93 \mathrm{~A}$ & $6,13 \mathrm{~A}$ \\
\hline $228+228$ & $6,88 \mathrm{~A}$ & $5,75 \mathrm{~A}$ & $4,94 \mathrm{~A}$ & $6,56 \mathrm{~A}$ \\
\hline $228+456$ & $6,38 \mathrm{~A}$ & $6,25 \mathrm{~A}$ & $5,06 \mathrm{~A}$ & $6,56 \mathrm{~A}$ \\
\hline$F(A)$ & $0,37^{\mathrm{ns}}$ & $0,66^{\mathrm{ns}}$ & $0,00^{n s}$ & $0,38^{n s}$ \\
\hline$F(B)$ & $0,41^{\text {ns }}$ & $0,94^{\mathrm{ns}}$ & $1,03^{n s}$ & $1,75^{\mathrm{ns}}$ \\
\hline$F(A \times B)$ & $1,14^{\mathrm{ns}}$ & $1,35^{\mathrm{ns}}$ & $0,79 \mathrm{~ns}$ & $1,12^{\mathrm{ns}}$ \\
\hline CV (\%) & 20,41 & 19,04 & 18,18 & 15,51 \\
\hline
\end{tabular}

Médias seguidas de mesma letra na coluna não diferem entre si ao nível de $5 \%$ de probabilidade pelo Teste de Tukey. Pelo teste F ns, não significativo; CV (\%), coeficiente de variação.

$\mathrm{Na}$ Tabela 8 verificou-se o desdobramento da interação entre as doses e formulações para a massa seca da parte aérea da cultivar IAC OL3. Entre as formulações de 2,4-D, verificou-se que o sal de amina causou redução no acúmulo de massa seca, quando comparado com o sal de colina. Entre as doses não houve diferença significativa para o sal de colina, mas para o sal de amina as doses sequenciais $(228+228$ e $228+456 \mathrm{~g}$ e.a. $\mathrm{ha}^{-1}$ ) promoveram redução de $27 \%$ para a massa seca da parte aérea das plantas. 
Tabela 7. Massa seca da parte aérea de plantas de amendoim 'IAC OL3', 'IAC 505', 'IAC OL4' e linhagem 870 submetidas às diferentes formulações e doses de 2,4-D.

\begin{tabular}{ccccc}
\hline Herbicidas (A) & IAC OL3 & L. 870 & IAC 505 & IAC OL4 \\
\hline Sal de colina & $14,17 \mathrm{~A}$ & $14,37 \mathrm{~A}$ & $14,35 \mathrm{~A}$ & $19,38 \mathrm{~A}$ \\
Sal de amina & $13,94 \mathrm{~A}$ & $13,72 \mathrm{~A}$ & $12,95 \mathrm{~A}$ & $18,83 \mathrm{~A}$ \\
\hline Doses (g e.a./ha) (B) & & & & \\
\hline 0 & $13,27 \mathrm{~A}$ & $16,27 \mathrm{~A}$ & $13,38 \mathrm{~A}$ & $15,76 \mathrm{~A}$ \\
228 & $15,81 \mathrm{~A}$ & $14,63 \mathrm{AB}$ & $12,98 \mathrm{~A}$ & $21,23 \mathrm{~A}$ \\
456 & $14,44 \mathrm{~A}$ & $13,39 \mathrm{AB}$ & $14,56 \mathrm{~A}$ & $20,92 \mathrm{~A}$ \\
910 & $15,18 \mathrm{~A}$ & $13,47 \mathrm{AB}$ & $13,77 \mathrm{~A}$ & $20,16 \mathrm{~A}$ \\
1370 & $14,01 \mathrm{~A}$ & $12,04 \mathrm{~B}$ & $14,31 \mathrm{~A}$ & $18,77 \mathrm{~A}$ \\
$114+228$ & $12,52 \mathrm{~A}$ & $14,89 \mathrm{AB}$ & $13,69 \mathrm{~A}$ & $19,40 \mathrm{~A}$ \\
$228+228$ & $14,69 \mathrm{~A}$ & $13,78 \mathrm{AB}$ & $13,61 \mathrm{~A}$ & $20,33 \mathrm{~A}$ \\
$228+456$ & $12,53 \mathrm{~A}$ & $13,89 \mathrm{AB}$ & $12,87 \mathrm{~A}$ & $16,28 \mathrm{~A}$ \\
\hline $\mathrm{F}(\mathrm{A})$ & $0,07 \mathrm{~ns}$ & $1,28 \mathrm{~ns}$ & $3,09 \mathrm{~ns}$ & $0,29 \mathrm{~ns}$ \\
\hline $\mathrm{F}(\mathrm{B})$ & $0,99 \mathrm{~ns}$ & $2,38^{*}$ & $0,27 \mathrm{~ns}$ & $2,05 \mathrm{~ns}$ \\
\hline $\mathrm{F}(\mathrm{AxB})$ & $2,39^{*}$ & $1,99 \mathrm{~ns}$ & $1,89 \mathrm{~ns}$ & $1,44 \mathrm{~ns}$ \\
\hline $\mathrm{CV}(\%)$ & 24,41 & 16,25 & 23,33 & 21,35 \\
\hline
\end{tabular}

Médias seguidas de mesma letra maiúscula na coluna não diferem entre si ao nível de 5\% de probabilidade pelo Teste de Tukey. Pelo teste $\mathrm{F},{ }^{*}$ significativo a $5 \%$ de probabilidade; ns, não significativo; CV (\%), coeficiente de variação.

Tabela 8. Desdobramento referente a massa seca da parte aérea da cultivar de amendoim 'IAC OL3' submetida às formulações e doses de 2,4-D.

\begin{tabular}{cccccc}
\hline Doses (g e.a./ha) & Sal de colina & \multicolumn{3}{c}{ Sal de amina } & $\mathrm{F}$ \\
\hline 0 & 17,99 & $\mathrm{Aa}$ & 13,63 & $\mathrm{Aa}$ & $1,76 \mathrm{~ns}$ \\
228 & 11,66 & $\mathrm{Aa}$ & 14,88 & $\mathrm{Aa}$ & $3,24 \mathrm{~ns}$ \\
456 & 13,25 & $\mathrm{Aa}$ & 15,63 & $\mathrm{Aa}$ & $0,97 \mathrm{~ns}$ \\
910 & 16,00 & $\mathrm{Aa}$ & 14,37 & $\mathrm{Aa}$ & $0,45 \mathrm{~ns}$ \\
1370 & 13,48 & $\mathrm{Aa}$ & 14,54 & $\mathrm{Aa}$ & $0,19 \mathrm{~ns}$ \\
$114+228$ & 15,91 & $\mathrm{Aa}$ & 13,47 & $\mathrm{Aa}$ & $1,01 \mathrm{~ns}$ \\
$228+228$ & 15,14 & $\mathrm{Aa}$ & 9,93 & $\mathrm{Bb}$ & $4,62^{*}$ \\
$228+456$ & 15,11 & $\mathrm{Aa}$ & 9,94 & $\mathrm{Bb}$ & $4,54^{*}$ \\
\hline $\mathrm{F}$ & $1,07 \mathrm{~ns}$ & & $2,31^{*}$ & & \\
\hline
\end{tabular}

Médias seguidas de mesma letra maiúscula na coluna e minúscula na linha não diferem entre si ao nível de $5 \%$ de probabilidade pelo Teste de Tukey. Pelo teste F, * significativo a $5 \%$ de probabilidade; ns, não significativo; CV (\%), coeficiente de variação. 
Tabela 9. Massa seca da raiz de plantas de amendoim 'IAC OL3', 'IAC 505', 'IAC OL4' e linhagem 870 submetidas às diferentes formulações e doses de 2,4-D.

\begin{tabular}{ccccc}
\hline Herbicidas (A) & IAC OL3 & L. 870 & IAC 505 & IAC OL4 \\
\hline Sal de colina & $4,94 \mathrm{~A}$ & $3,84 \mathrm{~A}$ & $4,18 \mathrm{~A}$ & $4,70 \mathrm{~A}$ \\
Sal de amina & $4,47 \mathrm{~A}$ & $4,12 \mathrm{~A}$ & $4,26 \mathrm{~A}$ & $4,72 \mathrm{~A}$ \\
\hline Doses (g e.a./ha) (B) & & & & \\
\hline 0 & $4,39 \mathrm{~A}$ & $4,90 \mathrm{~A}$ & $5,17 \mathrm{~A}$ & $4,91 \mathrm{ABC}$ \\
228 & $5,53 \mathrm{~A}$ & $4,85 \mathrm{~A}$ & $4,61 \mathrm{AB}$ & $4,94 \mathrm{ABC}$ \\
456 & $5,17 \mathrm{~A}$ & $3,47 \mathrm{AB}$ & $4,30 \mathrm{AB}$ & $4,77 \mathrm{ABC}$ \\
910 & $5,00 \mathrm{~A}$ & $3,63 \mathrm{AB}$ & $3,46 \mathrm{AB}$ & $3,98 \mathrm{BC}$ \\
1370 & $3,01 \mathrm{~A}$ & $2,80 \mathrm{~B}$ & $3,35 \mathrm{~B}$ & $3,58 \mathrm{C}$ \\
$114+228$ & $5,66 \mathrm{~A}$ & $4,23 \mathrm{AB}$ & $4,89 \mathrm{AB}$ & $5,22 \mathrm{AB}$ \\
$228+228$ & $4,49 \mathrm{~A}$ & $3,65 \mathrm{AB}$ & $4,07 \mathrm{AB}$ & $5,43 \mathrm{~A}$ \\
$228+456$ & $4,36 \mathrm{~A}$ & $4,32 \mathrm{AB}$ & $3,93 \mathrm{AB}$ & $4,87 \mathrm{ABC}$ \\
\hline $\mathrm{F}(\mathrm{A})$ & $1,23 \mathrm{~ns}$ & $1,12 \mathrm{~ns}$ & $0,07 \mathrm{~ns}$ & $0,01 \mathrm{~ns}$ \\
\hline $\mathrm{F}(\mathrm{B})$ & $1,98 \mathrm{~ns}$ & $3,78^{\star *}$ & $2,68^{*}$ & $3,83^{* *}$ \\
\hline $\mathrm{F}(\mathrm{AxB})$ & $0,90 \mathrm{~ns}$ & $0,55 \mathrm{~ns}$ & $2,47^{*}$ & $0,77 \mathrm{~ns}$ \\
\hline $\mathrm{CV}(\%)$ & 36,24 & 26,45 & 26,57 & 19,12 \\
\hline
\end{tabular}

Médias seguidas de mesma letra maiúscula na coluna não diferem entre si ao nível de $5 \%$ de probabilidade pelo Teste de Tukey. Pelo teste $\mathrm{F},{ }^{* *}$ significativo a $1 \% ;{ }^{*}$ significativo a $5 \%$ de probabilidade; ns, não significativo; CV (\%), coeficiente de variação.

Tabela 10. Desdobramento referente a massa seca da raiz da cultivar de amendoim IAC 505 submetida às diferentes formulações e doses de 2,4-D.

\begin{tabular}{cclclc}
\hline Doses (g e.a./ha) & Sal de colina & & Sal de amina & & $\mathrm{F}$ \\
\hline 0 & 5,72 & $\mathrm{Aa}$ & 4,62 & $\mathrm{Aa}$ & $1,93 \mathrm{~ns}$ \\
228 & 5,16 & $\mathrm{Aa}$ & 4,07 & $\mathrm{Aa}$ & $1,89 \mathrm{~ns}$ \\
456 & 5,21 & $\mathrm{Aa}$ & 3,39 & $\mathrm{ABb}$ & $5,29^{\star}$ \\
910 & 3,33 & $\mathrm{ABa}$ & 3,59 & $\mathrm{Aba}$ & $0,11 \mathrm{~ns}$ \\
1370 & 3,20 & $\mathrm{Ba}$ & 3,49 & $\mathrm{Aba}$ & $0,13 \mathrm{~ns}$ \\
$114+228$ & 5,52 & $\mathrm{Aa}$ & 4,27 & $\mathrm{Aa}$ & $2,46 \mathrm{~ns}$ \\
$228+228$ & 4,16 & $\mathrm{ABa}$ & 3,98 & $\mathrm{Aa}$ & $0,05 \mathrm{~ns}$ \\
$228+456$ & 4,86 & $\mathrm{Aa}$ & 3,01 & $\mathrm{Bb}$ & $5,47^{*}$ \\
\hline $\mathrm{F}$ & $3,99^{\star *}$ & & $3,16^{* *}$ & &
\end{tabular}

Médias seguidas de mesma letra maiúscula na coluna e minúscula na linha não diferem entre si ao nível de $5 \%$ de probabilidade pelo Teste de Tukey. Pelo teste $\mathrm{F},{ }^{* *}$ significativo a $1 \%$; * significativo a $5 \%$ de probabilidade; ns, não significativo. 
Tabela 11. Massa seca total de plantas de amendoim 'IAC OL3', 'IAC 505', 'IAC OL4' e linhagem 870 submetidas às diferentes formulações e doses de 2,4-D.

\begin{tabular}{ccccc}
\hline Herbicidas $(\mathrm{A})$ & IAC OL3 & L. 870 & IAC 505 & IAC OL4 \\
\hline Sal de colina & $19,11 \mathrm{~A}$ & $18,21 \mathrm{~A}$ & $18,53 \mathrm{~A}$ & $24,08 \mathrm{~A}$ \\
Sal de amina & $18,41 \mathrm{~A}$ & $17,84 \mathrm{~A}$ & $17,21 \mathrm{~A}$ & $23,55 \mathrm{~A}$ \\
\hline Doses (g e.a./ha) (B) & & & & \\
\hline 0 & $17,66 \mathrm{~A}$ & $19,53 \mathrm{~A}$ & $18,55 \mathrm{~A}$ & $20,67 \mathrm{~A}$ \\
228 & $21,34 \mathrm{~A}$ & $21,12 \mathrm{~A}$ & $17,59 \mathrm{~A}$ & $26,16 \mathrm{~A}$ \\
456 & $19,61 \mathrm{~A}$ & $16,86 \mathrm{AB}$ & $18,86 \mathrm{~A}$ & $25,69 \mathrm{~A}$ \\
910 & $20,18 \mathrm{~A}$ & $17,10 \mathrm{AB}$ & $17,24 \mathrm{~A}$ & $24,14 \mathrm{~A}$ \\
1370 & $17,02 \mathrm{~A}$ & $14,84 \mathrm{~B}$ & $17,65 \mathrm{~A}$ & $22,35 \mathrm{~A}$ \\
$114+228^{* * *}$ & $18,18 \mathrm{~A}$ & $19,11 \mathrm{AB}$ & $18,58 \mathrm{~A}$ & $24,62 \mathrm{~A}$ \\
$228+228^{* * *}$ & $19,18 \mathrm{~A}$ & $17,43 \mathrm{AB}$ & $17,68 \mathrm{~A}$ & $25,76 \mathrm{~A}$ \\
$228+456^{* * *}$ & $16,90 \mathrm{~A}$ & $18,21 \mathrm{AB}$ & $16,81 \mathrm{~A}$ & $21,15 \mathrm{~A}$ \\
\hline $\mathrm{F}(\mathrm{A})$ & $0,37 \mathrm{~ns}$ & $0,29 \mathrm{~ns}$ & $1,95 \mathrm{~ns}$ & $0,24 \mathrm{~ns}$ \\
\hline $\mathrm{F}(\mathrm{B})$ & $0,96 \mathrm{~ns}$ & $4,01^{* *}$ & $0,29 \mathrm{~ns}$ & $1,95 \mathrm{~ns}$ \\
\hline $\mathrm{F}(\mathrm{AxB})$ & $1,48 \mathrm{~ns}$ & $1,89 \mathrm{~ns}$ & $2,20 \mathrm{~ns}$ & $1,36 \mathrm{~ns}$ \\
\hline $\mathrm{CV}(\%)$ & 24,42 & 15,02 & 21,18 & 18,40 \\
\hline
\end{tabular}

Médias seguidas de mesma letra maiúscula na coluna não diferem entre si ao nível de $5 \%$ de probabilidade pelo Teste de Tukey. Pelo teste $\mathrm{F}$, ** significativo a 1\% de probabilidade; ns, não significativo; CV (\%), coeficiente de variação.

Para a massa seca radicular da cultivar IAC 505 (Tabela 10), observou-se interação entre as doses e formulações de 2,4-D. Entre as formulações, constatou-se que o sal de amina causou maior redução na massa seca que o sal de colina na dose única (456 g e.a. ha-1) e na sequencial $\left(228+456 \mathrm{~g}\right.$ e.a. ha $\left.{ }^{-1}\right)$. Entre as doses, verificou-se que para o sal de colina, a maior dose em aplicação isolada (1370 g e.a. ha-1) causou $44 \%$ de redução na massa seca das raízes. Para o sal de amina, a maior dose sequencial $\left(228+456\right.$ g e.a. ha-1 ${ }^{-1}$ causou a maior redução $(34 \%)$ na massa seca radicular. Além da aplicação sequencial, também se observou que as maiores doses isoladas (456; 910 e $1370 \mathrm{~g}$ e.a. ha-1) foram prejudiciais para essa cultivar, causando reduções de $27 \%, 22 \%$ e $24 \%$, respectivamente.

Quando se analisa a massa seca total, não se observa diferenças entre as cultivares (IAC 505, IAC OL3 e IAC OL4). Para a linhagem 870, observou-se que a maior dose (1370 g e.a. ha-1 ${ }^{-1}$ causou redução de $24 \%$ quando comparada com a testemunha (Tabela 11). Essa linhagem foi muito sensível ao uso de 2,4-D, principalmente quando submetida às maiores 
doses. Blanchett et al. (2017), testando doses de 2,4-D, não constataram redução na massa seca do amendoim até a dose de $1066 \mathrm{~g}$ e.a. ha-1.

\section{Conclusões}

Independente da formulação (sal de colina e sal de amina), o uso do 2,4-D nas doses isoladas (228 e $456 \mathrm{~g}^{\mathrm{e}}$.a. ha- ${ }^{-1}$ ) e/ou sequencial $(114+128$; 228+228 g e.a.ha-1 ${ }^{-1}$ não foram fitotóxicas, enquanto as doses de 1370; $910 \mathrm{e}$ 228+456 g e.a.ha-1 foram fitotóxicas para as cultivares IAC 505, IAC OL3 e IAC OL4 e a linhagem 870. As plantas de amendoim sob o efeito do 2,4-D, independente da formulação, apresenta comportamento morfológico diferencial entre os genótipos avaliados. 


\section{Referências}

AGOSTINHO; GRAVENA; ALVES; SALGADO; MATTOS. (2006). The effect of cultivar on critical periods of weed control in peanuts. Peanut Science, 33, 29-35.

ALVINO; GRICIO; SAMPAIO; GIROTTO; FELIPE; JUNIOR; BUENO; BOSQUÉ; LIMA. Interferência e controle de plantas daninhas nas culturas agrícolas. Revista Científica Eletrônica de Agronomia, vol. 10, n. 20, p. 3, 2011.

ANONYMOUS (2013) Enlist. Weed Control System Technical Bulletin. Indianapolis, IN: Dow AgroSciences. $13 p$

BARBOSA J. C.; MALDONADO JÚNIOR W. Experimentação agronômica e agroestat: sistema para análise estatística de ensaios agronômicos. Jaboticabal: Faculdade de Ciências Agrárias e Veterinária, Unesp, 2015. 396 p.

BLANCHETT; GREY; PROSTKO; WEBSTER; VENCILL. The Effect of 2, 4Dichlorophenoxyacetic Acid (2,4-D) on Peanut when Applied During Vegetative Growth Stages. Peanut Science, v. 44, n. 1, p. 53-59, 2017.

CONAB. Companhia Nacional de Abastecimento. Acompanhamento da Safra Brasileira de Grãos. Disponível em: http://www.conab.gov.br. Acesso em: 13 de Julho de 2021. 2021a.

CONAB. Companhia Nacional de Abastecimento. Safras 1976/77 a 2020/21: série histórica. Brasília, DF. Disponível em: http://www.conab.gov.br/conteudos.php?a=1252. Acesso em: 02 de Julho de 2021. $2021 b$.

EWRC - EUROPEAN WEED RESEARCH COUNCIL. Report of 3rd and 4 rd meetings of EWRC. Cittee of methods in weed research. Weed Research. v. 4. p. $88,1984$.

GROSSMANN (2010). Auxin herbicides: current status of mechanism and mode of action. Pest Manag Sci 66:113-120

GUNSOLUS; CURRAN. (1991) Herbicide Mode of Action and Injury Symptoms, North Central Regional Extension Publication 377. Washington, DC: U.S. Department of Agriculture Cooperative Extension Services.

JOHNSON; FISHER; JORDAN; EDMISTEN; STEWART; YORK. (2012). Cotton, peanut, and soybean response to sublethal rates of dicamba, glufosinate, and 2, 4-D. Weed Technology, v. 26, n. 2, p. 195-206, 2012.

LEON; FERRELL; BRECKE. Impact of exposure to 2, 4-D and dicamba on peanut injury and yield. Weed Technology, v. 28, n. 3, p. 465-470, 2014. 
LI; TANK; KENNEDY; ZHANG; DOWNER; OUSE; LIU (2013). Enlist Duo herbicide: a novel 2,4-D plus glyphosate premix formulation with low potential for off-target movement. in Pesticide Formulation and Delivery Systems. Volume 32. Innovating Legacy Products for New Uses. West Conshohocken, PA: ASTM International

MAPA. Ministério da Agricultura, Pecuária e Abastecimento. Cultura com Suporte Fitossanitário Insuficiente. Registro de agrotóxicos para culturas com suporte fitossanitário insuficiente. Manual de procedimentos. Instrução normativa conjunta 01, de 16 de junho de 2014. 2015. 24p. Disponível em: http://www.agricultura.gov.br/assuntos/insumos-agropecuarios/insumosagricolas/agrotoxicos/culturas-com-suporte-fitossanitario-insuficiente-csfi. Acesso em: 06/07/2021.

NEPOMUCENO; ALVES; DIAS; CARDOZO; PAVANI; (2007). Efeito da época de semeadura nas relações de interferência entre uma comunidade infestante e a cultura do amendoim. Planta Daninha, 25, 481-488.

OLIVEIRA JÚNIOR; et al. Efeito de subdoses de 2,4-D na produtividade de uva Itália e suscetibilidade da cultura em função de seu estádio de desenvolvimento. Engenharia Agrícola, Jaboticabal, v. 27, n. esp., p. 3540, 2007.

Peterson; McMaster; Riechers; Skelton; Stahlman (2016). 2,4-D Past, Present,and Future: A Review Weed Technol 30:303-345

PITELLI. A. Competição e controle de plantas daninhas em áreas agrícolas. Série TECNICA IPEF, v.4, n.12, 1-24 p, 1987.

PITELLI. Interferência de Plantas daninhas Culturas Agrícolas. Informe Agropecuário, Belo Horizonte, v.11, p.16-25, 1985.

RODRIGUES; ALMEIDA. (2011). Guia de herbicidas. 6 ed. Londrina, Paraná. 697 p.

SONG, Y. Insight into the mode of action of 2, 4- dichlorophenoxyacetic acid (2, 4-D) as an herbicide. Journal of Integrative Plant Biology, v.56, n.2, p.106-113, 2014.

SOSNOSKIE; CULPEPPER; BRAXTON; RICHBURG (2015) Evaluating the volatility of three formulations of 2,4-D when applied in the field. Weed Technol 29:177-184

USDA. United States Departamento of Agriculture. World Agricultural Production. Foreign Agricultural Service (Circular Series WAP 06-21), Junho 2021. Disponivel

em: https://apps.fas.usda.gov/psdonline/circulars/production.pdf. Acesso em: 02/07/2021.

YAMAUTI; ALVES; NEPOMUCENO; \& MARTINS. (2010). Adubação e o período anterior à interferência das plantas daninhas na cultura do amendoim. Planta Daninha, 28, 1031-1039. 